\title{
Twin, Aligned Protoplanetary Disks around the Components of the Young Binary System L1551 IRS5
}

\author{
José M. Torrelles \\ Institut d'Estudis Espacials de Catalunya (IEEC/CSIC) and Instituto \\ de Ciencias del Espacio (CSIC), Gran Capità 2, 08034 Barcelona, Spain
}

Luis F. Rodríguez

Instituto de Astronomía, UNAM, Apdo. Postal 72-3 (Xangari), 58089 Morelia, México

\author{
Guillem Anglada \\ Instituto de Astrofísica de Andalucía (CSIC), Apdo. Correos 3004, \\ 18080 Granada, Spain
}

\begin{abstract}
VLA $7 \mathrm{~mm}$ continuum observations carried out by Rodríguez et al. (1998) with $0.06^{\prime \prime}$ resolution toward L1551 IRS5 have shown two resolved dust sources. Both sources are elongated with almost the same orientation, similar flux densities, and similar angular sizes, being interpreted by these authors as a system of twin, aligned protoplanetary disks of $10 \mathrm{AU}$ radius around the components of a young binary of $50 \mathrm{AU}$ separation. These protoplanetary disks, the smaller ones ever imaged at $\mathrm{mm}$ wavelengths, are about a factor of ten smaller than disks around isolated stars, but have enough mass to form solar systems similar to our own. The fact that the disks are aligned with each other argues in favor for long-lived planetary systems in this binary. We all look forward to the future development of interferometers such as the EVLA, SMA, and ALMA in order to characterize the physical conditions of these associated protoplanetary disks by detecting spatially resolved molecular line emission. With these upcoming interferometers it will be possible to make great strides forward in our knowledge of the star formation processes.
\end{abstract}

\section{Introduction}

Several days before the beginning of the IAU Symposium 200 on the "Formation of Binary Stars" in Postdam, we received an e-mail from Bob Mathieu and Hans Zinnecker with some indications to clarify our role as presenters of the "featured objects" talks. According to that message, we should concentrate in our talk on: (1) what has actually been observed in the object, and (2) the nature and properties of the object, leaving broad implications for binary formation to the review speakers. We would like to thank Bob and Hans for their helpful general comments to arrange the different talks for the Symposium, and following their indications we have divided this paper on L1551 IRS5 in these two main sections 


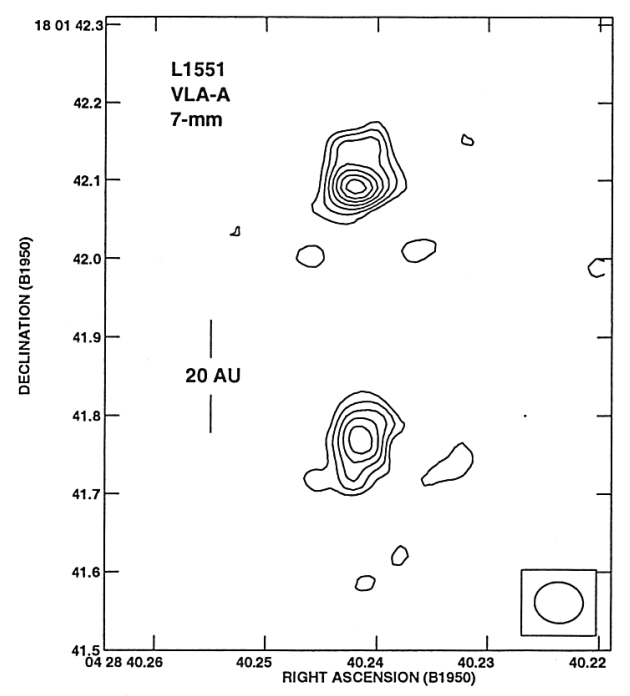

Figure 1. VLA $7 \mathrm{~mm}$ sources in L1551 IRS5 (Rodríguez et al. 1998).

$(\S \S 2,3)$. However, since Bob at the end of our oral presentation asked us for some possible implications on binary formation based on our results, we also present a short section $(\S 4)$ with some of these possible implications and future work, in particular for the formation of planetary systems in L1551 IRS5.

\section{What Has Actually Been Observed in L1551}

The outflow in L1551 (Snell, Loren, \& Plambeck 1980) was, together with Cepheus A (Rodríguez, Ho, \& Moran 1980), the first bipolar molecular outflow detected around young stellar objects (YSOs). The powering source, first detected at infrared wavelengths (IRS5) (Strom, Strom, \& Vrba 1976), is embedded in a high-density molecular condensation with dimensions of about $10^{4} \mathrm{AU}$ (Torrelles et al. 1983). The luminosity of this source is $\simeq 30 \mathrm{~L}_{\odot}$, corresponding to a low-mass young star.

Very Large Array (VLA) $2 \mathrm{~cm}$ continuum observations show two compact sources toward L1551 IRS5 separated by $\simeq 0.3^{\prime \prime}$. These radio sources were interpreted as either a protobinary system separated by $50 \mathrm{AU}$ (Bieging \& Cohen 1985 ) or the inner ionized walls of a toroid surrounding a single star (Rodríguez et al. 1986).

More recently, Looney et al. (1997) through BIMA $2.7 \mathrm{~mm}$ continuum observations with $\simeq 0.5^{\prime \prime}$ resolution detected an elongated dust emission structure toward L1551 IRS5, arguing for the presence of two unresolved circumstellar disks around a binary system. In addition, these authors also argued for the presence of a circumbinary structure and an envelope of $\simeq 150 \mathrm{AU}$ and $3000 \mathrm{AU}$ size, respectively.

Finally, $7 \mathrm{~mm}$ continuum observations made with the VLA with $\simeq 0.06^{\prime \prime}$ resolution $(\simeq 7 \mathrm{AU}$ at the source distance), show two resolved sources (Figure 
1), both elongated with almost the same orientation north-south, similar flux densities and similar angular sizes (Rodríguez et al. 1998).

\section{Nature and Properties of the Sources in L1551}

These two $7 \mathrm{~mm}$ sources have been interpreted by Rodríguez et al. (1998) as a system of "twin, aligned protoplanetary disks" in a binary star, based on two main arguments: (1) The 7 and $2.7 \mathrm{~mm}$ flux densities are too large to be accounted by free-free emission from ionized gas. The observed flux densities at these frequencies are most probably produced by heated dust. (2) The $7 \mathrm{~mm}$ sources are both elongated north-south, perpendicular to the observed centimeter emission which traces the ionized outflow, and disk and jet are expected to show a relative perpendicular orientation. Furthermore, two jets have been recently reported toward L1551 IRS5 (Itoh et al. 2000; Fridlund \& Liseau 1998; Hartigan et al. 2000; see Itoh in these proceedings), each probably originating from a different star of the binary system.

The two protoplanetary disks are separated by $\simeq 50 \mathrm{AU}$, have radii of $\simeq 10$ $\mathrm{AU}$, and masses have been estimated to be $\simeq 0.05 \mathrm{M}_{\odot}$ (Rodríguez et al. 1998).

In Figure 2 we show a "cartoon" made by Oliver Lay illustrating different aspects observed in the central region of L1551 IRS5. All the components shown in this "cartoon" (with the exception of the circumbinary disk, whose structure has not been imaged yet, see Looney et al. 1997) have been already detected.

\section{General Comments}

The two protoplanetary disks around the young binary L1551 IRS5 are the smaller ones ever imaged at $\mathrm{mm}$ wavelengths around YSOs. In spite of the small size of these disks (a factor of ten smaller than other disks found around isolated stars), both disks have enough mass to form solar systems similar to our own. Furthermore, the fact that the disks are aligned with each other argues in favor for long-lived planetary systems in this binary star system (see Jensen, Donar, $\&$ Mathieu 2000 in the poster proceedings of this Symposium for a discussion of aligned disks in pre-main sequence binaries and their implications).

VLA continuum observations have been proven to be an excellent tool to discover new young binary stars, particularly the close systems. Recently, binary sources with angular separation below one arcsec, and with the components apparently in different evolutive stages, have been discovered in association with the SVS 13 (Anglada, Rodríguez, \& Torrelles 2000) and YLW 15 (Girart, Rodríguez, \& Curiel 2000) stars. Partial, but useful kinematical information can be obtained from VLA and VLBA observations of $\mathrm{H}_{2} \mathrm{O}$ maser spots in protoplanetary disks at radii of a few of tens AU (e.g., Torrelles et al. 1998). However, in order to properly characterize the physical conditions of the associated protoplanetary disks in YSOs (kinematics, temperature profile, chemistry composition, dust to gas ratio, evolution, etc.) we need to detect spatially resolved molecular line emission from these disks. This is the reason why we all look forward to the new development of interferometers such as the Expanded Very Large Array (EVLA; NRAO), the Submillimeter Array (SMA; HarvardSmithsonian Center for Astrophysics) and the Atacama Large Millimeter Array 


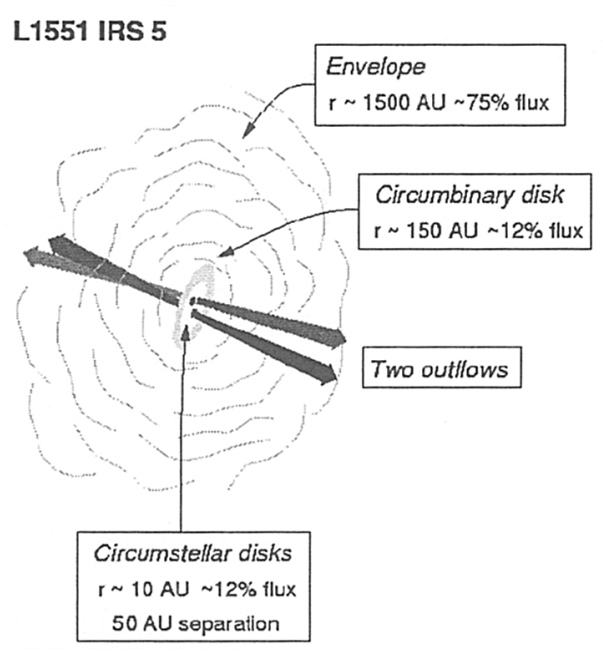

Figure 2. "Cartoon" of the inner region of L1551 drawn by Oliver Lay.

(ALMA; NRAO-European Consortium). This will help to make great strides forward in our knowledge of the star formation processes, in general, and on the origin of our own Solar System, in particular.

\section{References}

Anglada, G., Rodríguez, L. F., \& Torrelles, J. M. 2000, submitted to ApJ Bieging, J. H., \& Cohen, M. 1985, ApJ, 289, L5

Fridlund, C. V. M., \& Liseau, R. 1998, ApJ, 499, L75

Girart, J. M., Rodríguez, L. F., \& Curiel, S. 2000, submitted to ApJ

Hartigan, P., Morse, J., Palunas, P., Bally, J., Devine, D. 2000, AJ, 119, 1872 Itoh, Y. et al. 2000, PASJ, 52, 81

Jensen, E. L., Donar, A. X., \& Mathieu, R. D. 2000, in Poster Proceedings of IAU Symposium 200, ed. Bo Reipurth \& Hans Zinnecker, 85

Looney, L. W., Mundy, L. G., \& Welch, W. J. 1997, ApJ, 484, L157

Rodríguez, L. F., Cantó, J., Torrelles, J. M., \& Ho, P. T. P. 1986, ApJ, 301, L25

Rodríguez, L. F. et al. 1998, Nature, 395, 355

Rodríguez, L. F., Ho, P. T. P., \& Moran, J. M. 1980, ApJ, 240, L149

Snell, R. L., Loren, R. B., \& Plambeck, R. L. 1980, ApJ, 239, L17

Strom, K. M., Strom, S. E., \& Vrba, F. J. 1976, AJ, 81, 320

Torrelles, J. M. et al. 1983, ApJ, 274, 214

Torrelles, J. M. et al. 1998, ApJ, 505, 756 